\title{
Factores cardiovasculares y renales asociados a hipertensión arterial en pediatría
}

Cardiovascular and kidney factors associated with arterial hypertension in pediatrics

\section{* Juan Rafael Guerrero Manueles, * Tania Soledad Licona Rivera. ***}

\section{RESUMEN}

Antecedentes: El aumento de la prevalencia de Hipertensión Arterial (HTA) en Pediatría se debe a factores de riesgo (FR) relacionados con el comportamiento.Objetivo: Determinar los FR Cardiovasculares y Renales asociados a HTA en Pediatría en el Instituto Hondureño de Seguridad Social- Hospital Regional del Norte (IHSS-HRN) y Diálisis de Honduras-San Pedro Sula (SPS) en el período de enero 2017 - junio 2018.Pacientes y Métodos: Estudio de Casos y Controles en pacientes de 29 días-17 años con 364 días de edad, nefropatas y/o cardiópatas y que no sean cardiópatas o nefropatas que tengan o no HTA que acudieron a la Consulta Externa de Cardiología Pediátrica y pacientes que estuvieron hospitalizados en la Emergencia de Pediatría, Sala de Unidad de Cuidados Intensivos (UCIP), Sala de Pediatría A y B del IHSS-HRN y Diálisis de Honduras-SPS. El tamaño de la muestra fue de 148 pacientes. Se le realizó Regresión Logística Binaria (RLB) para determinar los FR.

Resultados: La prevalencia de HTA en el IHSS-HRN fue de $0.24 \%$ y $2.7 \%$ en Diálisis de Honduras-SPS. Los FR identificados en el análisis de RLB fueron: mayor de 10 años, masculino, sedentarismo, consumo de alcohol y cigarrillos, antecedentes familiares de HTA y antecedentes personales de enfermedades renales. Según los resultados de Estadísticas de Wald con valor de $\mathrm{P}<0.05$ indicaron que

\footnotetext{
${ }^{*}$ Residente de 3er año del Postgrado de Pediatría, UNAH-VS.

${ }^{* *}$ Cardiólogo Pediatra del IHSS-HRN.

***Docente del Departamento de Medicina Clínica Integral EUCS UNAH-VS.

Dirigir correspondencia: marlon_alexander07@yahoo.com

Recibido: 10 de Junio de 18 Aceptado: 10 Septiembre de 2018
}

estos resultados se pueden generalizar a la población. Conclusiones: Los FR para HTA en pediatría están relacionados con el comportamiento y pueden ser prevenibles y modificables desde etapas tempranas de la infancia.

\section{PALABRAS CLAVE}

Hipertensión, Factores de Riesgo, Niños.

\section{ABSTRACT}

Arterial Hypertension (HTA) is the cause of at least $45 \%$ of deaths from acquired heart diseases and $51 \%$ of deaths from cardiovascular diseases. The increase in the prevalence of hypertension is due to risk factors related to behavior.

Objective: To determine the cardiovascular and renal factors associated to arterial hypertension in pediatrics in the Honduran Institute of Social Security (IHSS-HRN) and Dialysis of Honduras-SPS in the period from January 2017 to June 2018.

Patients and Methods: Study of Cases and Controls in patients of 29 days-17 years with 364 days of age, nephropaths and / or cardiologists and who are not cardiologists or nephropaths with or without HTA who attended the Outpatient Consultation of Pediatric Cardiology and patients who were hospitalized in the Pediatric Emergency, Intensive Care Unit Room (PICU), Pediatrics Room A and B of the IHSS-HRN and Dialysis of Honduras-SPS. The sample size was 74 cases and 74 controls. Binary Logistic Regression was performed to determine the Risk Factors. Results: The prevalence of hypertension in the IHSS-HRN was $0.24 \%$ and $2.7 \%$ in Dialysis of Honduras-SPS. 
The risk factors identified in the study were: age over 10 years, male sex, sedentary lifestyle, alcohol and cigarette consumption. adding salt to food, family history of hypertension and personal history of kidney disease. According to the results of Wald statistics with a value of $P<0.05$, they indicated that these results can be generalized to the population. Conclusions: The risk factors for hypertension in pediatrics are related to behavior and can be preventable and modifiable from early childhood.

\section{KEYWORDS}

Hypertension, Risk Factors, Children.

\section{INTRODUCCIÓN}

La definición más aceptada de HTA en pediatría es la propuesta por la Academia Americana de Pediatría (AAP) 2017. Se define HTA en niños y adolescentes cuando los valores de PA sistólica y/o diastólica (PAS y/o PAD) se encuentran de forma repetida, en tres o más en ocasiones separadas, igual o por encima del percentil 90 específica para la edad, sexo y talla, según las tablas de normalización(1) . A nivel global las enfermedades cardiovasculares son responsables de aproximadamente 17 millones de muertes por año, casi un tercio del total(2). Entre ellas, las complicaciones de la Hipertensión Arterial (HTA) causan anualmente 9.4 millones de muertes ${ }^{(3)}$. La HTA es la causa de por lo menos $45 \%$ de las muertes por cardiopatías y $51 \%$ por enfermedades cardiovasculares $^{(4)}$. Se estima que uno de cuatro adultos sufre de HTA, una enfermedad que es el principal factor de riesgo para las siguientes enfermedades: arterioesclerótica, cerebrovascular, cardiaca isquémica y la segunda causa de insuficiencia renal crónica en adultos ${ }^{(5)}$. La prevalencia de HTA es mayor entre los adolescentes que entre los niños más pequeños (4). Un estudio realizado en la ciudad de El Progreso, Honduras demostró que la prevalencia de prehipertensión en la edad comprendida entre 18-27 años fue de $65 \%{ }^{(6)}$ y otro estudio realizado en la Col. Miraflores, Tegucigalpa la prevalencia fue de HTA $32.7 \%$ entre la población estudiada(7). Además varios estudios longitudinales han demostrado que las alteraciones en la PA en la infancia y adolescencia con frecuencia se traducen en HTA en edades adultas por lo que es importante la búsqueda de esta condición en edad pediátrica mediante estudios de investigación (8-14).

El objetivo de este estudio es contribuir al conocimiento de los Factores Cardiovasculares y Renales implicados en la HTA en niños y adolescentes. Una mayor comprensión de los factores de riesgo, permitirá un abordaje más holístico de la HTA en la población pediátrica, así mismo implementar algunas estrategias de prevención cardiovascular las cuales solo eran destinadas a la población adulta, dado el gran impacto y relevancia que tiene todas las repercusiones cardiovasculares a corto y largo plazo, esto nos permitirá generar políticas públicas que promuevan estilos de vida saludable en la población infantil.

\section{PACIENTES Y MÉTODOS}

Se realizó un estudio de Casos y Controles, con enfoque cuantitativo, diseño no experimental, corte transversal, alcance analítico y descriptivo. El período comprendido del estudio fue del 1 enero 2017 al 30 de Junio 2018. El universo lo conformaron los pacientes pediátricos de 29 días-17 años con 364 
niños afroamericanos e hispanos en comparación con los niños blancos no hispanos, con tasas más altas entre los días de edad, nefrópatas y/o cardiópatas y que no sean cardiópatas y/o nefrópatas que tengan o no HTA que acudieron a la Consulta Externa de Cardiología Pediátrica y pacientes que estuvieron hospitalizados en la Emergencia de Pediatría, Sala de Unidad de Cuidados Intensivos (UCIP), Sala de Pediatría A y B del Instituto Hondureño de Seguridad Social (IHSS-HRN) y Diálisis de Honduras (SPS), que tengan o no HTA. El tamaño de la muestra fue de 148 pacientes distribuidos en 74 casos y 74 controles apareados por sexo y edad. El método de muestreo fue no probabilístico. Los criterios de inclusión para los casos y controles fueron Todos los pacientes pediátricos de 29 días-17 años con 364 días de edad, nefropatas cardiópatas que tengan o no HTA que acudieron a la Consulta Externa de Cardiología Pediátrica, que ingresaron a la Emergencia de Pediatría, Sala de Unidad de Cuidados Intensivos (UCIP), Sala de Pediatría A y B del IHSSHRN, acudieron a Diálisis de Honduras (SPS) y que previa autorización de los padres hayan aceptado participar en la investigación.

Se excluyeron todos los pacientes que no desearon participar en el estudio, menores de 29 días y 18 años cumplidos, y que no estuvieran afiliados al IHSS-HRN y Diálisis de Honduras (SPS). Las hipótesis nulas fueron que los pacientes menores de 10 años de edad, sexo femenino tienen mayor riesgo de desarrollar HTA y el consumo de café y los antecedentes familiares de HTA, enfermedades renales y cardiacas no se asocian con HTA en Pediatría. Para recolectar los datos se utilizó un cuestionario tipo entrevista que contenía 35 preguntas divido en 4 secciones, en la sección I (Datos Generales),
Sección II (Datos Familiares), Sección III (Hábitos) y Sección IV (Antecedentes Médicos Personales). El instrumento se aplicó a los casos y controles. Se determinó la evidencia relacionada con la validez de contenido del instrumento, que es el grado en que la medición representa el concepto medido, mediante el juicio de expertos y aplicación de la prueba piloto, que evaluaron $y$ analizaron las preguntas del cuestionario, basándose en una matriz de adecuación que contiene los criterios: induce a una respuesta determinada, sencillez en la redacción, ambigüedad, relación con el tema, claridad, adaptada al contexto de aplicación sin propuesta de cambios por parte de los expertos, el instrumento antes de ser aplicado fue aprobado por el asesor metodológico. El análisis de datos se realizó en el programa SPSS versión 23.0. Para determinar la confiabilidad de los resultados finales de la investigación se aplicó la Prueba de Durbin-Watson la cual nos indicó que se cumplió el supuesto de independencia de errores, así mismo, se aplicaron Estadísticas de Colinealidadpara confirmar que no hubiesen multicolinealidades independientes. entre las variables Para el análisis multivariado con el objetivo de determinar la distribución de los factores de riesgo cardiovascular y renal asociados a Hipertensión Arterial en Pediatría se aplicó el modelo de Regresión Logística Binaria. Los gráficos se realizaron en el programa Microsoft Excel 2017. Debido a que se trata de una investigación no experimental, en la que no existe riesgo alguno para los participantes, es un estudio de categoría I. RESULTADOS

La prevalencia de HTA en la población estudiada en el IHSS-HRN fue de $0.2 \%$ y $2.7 \%$ en Diálisis de Honduras-SPS. 
En relación a las Características Sociodemográficas los casos corresponden al $50 \% \quad(74)$, donde $45 \quad(60.8 \%)$ son masculinos y 29 (39.2\%) son femeninos. Los controles corresponden al 50\% (74), donde $37(50.0 \%)$ son masculinos y 37 $(50.0 \%)$ fueron femeninas. El rango de edad de 11-15 años en los pacientes con HTA representa el $43.2 \%$ (32), encontrándose una media de edad de 11.3 años, mediana de 12.5 años $y$ moda de 13años en los casos. (Ver Gráfico No. 1).

Gráfico No. 1: Distribución por Edad de la Población Estudiada.

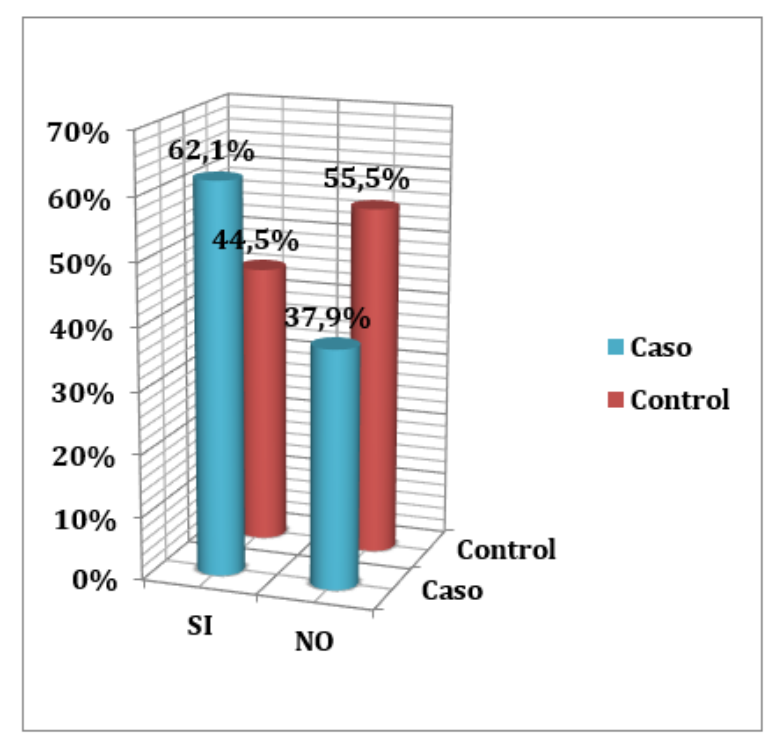

Fuente: Estudio de Factores de Riesgo Cardiovascular y Renal asociados a HTA en Pediatría. De los pacientes con HTA el $62.1 \% \quad(46)$ consumían café. (Ver Gráfico No. 2)

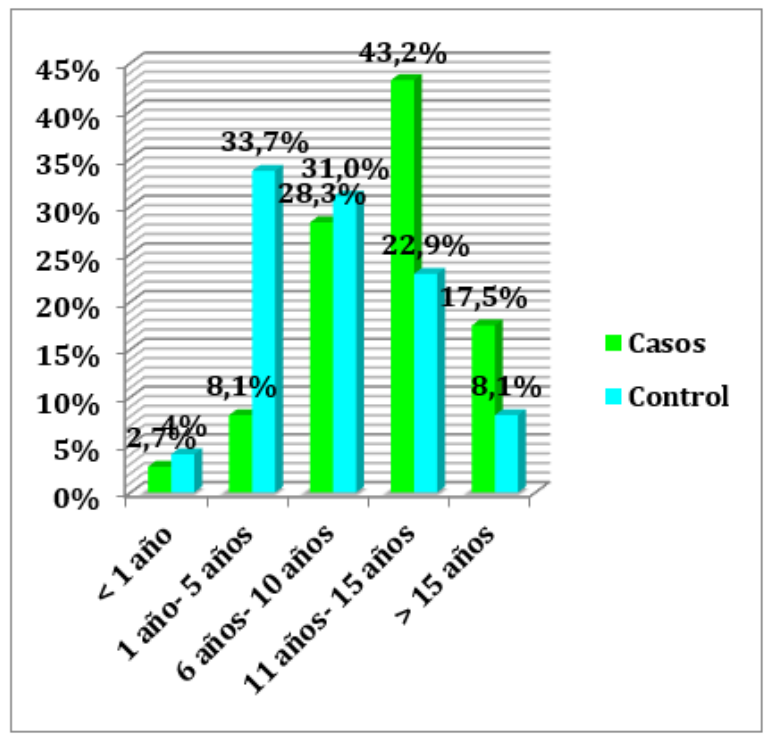

Fuente: Estudio de Factores de Riesgo Cardiovascular y Renal asociados a HTA en Pediatría. De los pacientes con HTA el $77.1 \%$ (57) no realizaban ejercicio. (Ver Gráfico No. $3)$.

Gráfico No. 3: Distribución de pacientes que Realizan Ejercicio en la Población Estudiada.

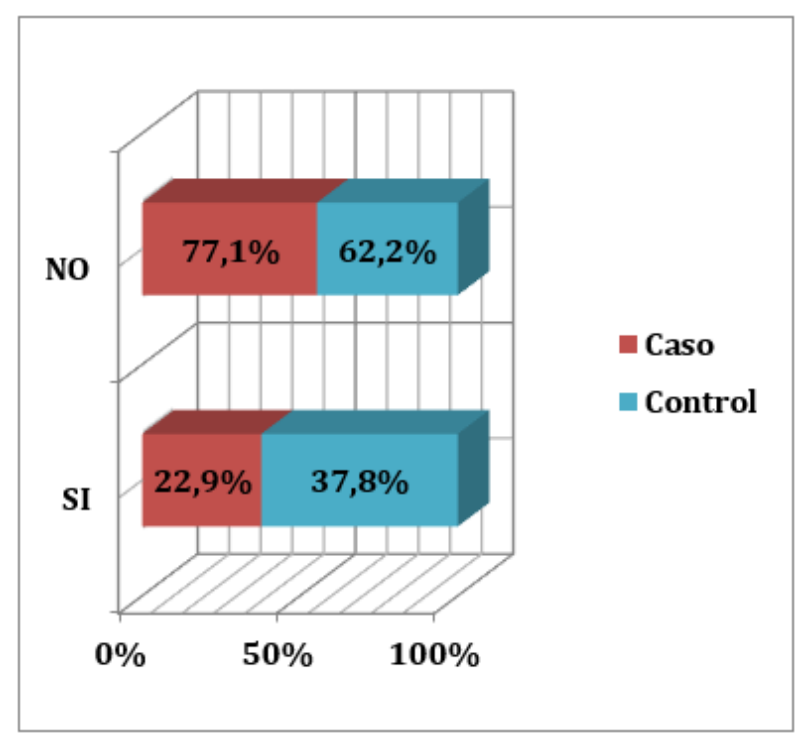


Fuente: Estudio de Factores de Riesgo Cardiovascular y Renal asociados a HTA en Pediatría.

De los pacientes con HTA el $64.8 \%$ (48) le agregaban pisca de sal a los alimentos. (Ver Gráfico No. 4).

Gráfico No. 4: Distribución de pacientes que Agregan Pisca de Sal a los Alimentos en la Población Estudiada.

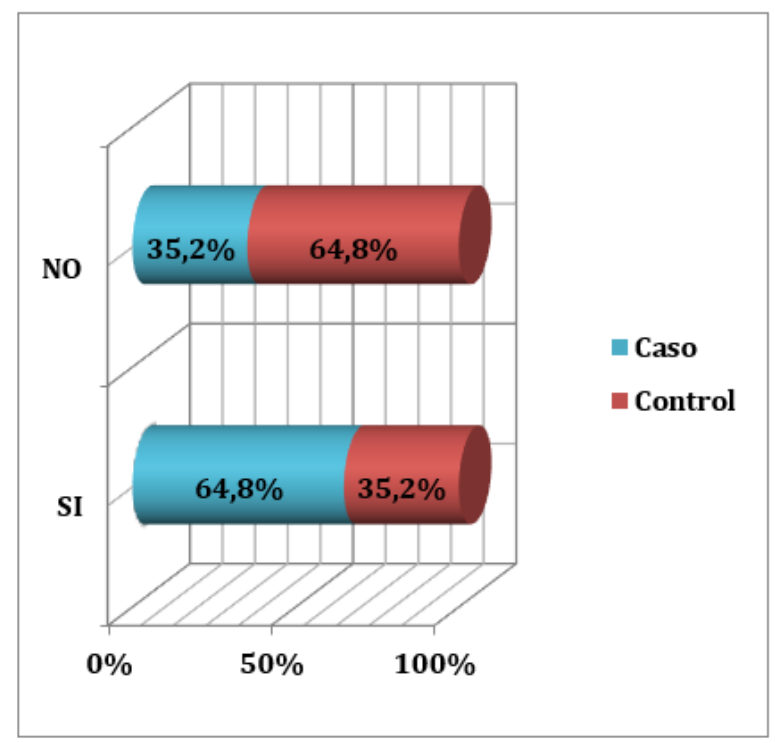

Fuente: Estudio de Factores de Riesgo Cardiovascular y Renal asociados a HTA en Pediatría.

De los pacientes con HTA al $35.8 \%$ (53), le tomaron ocasionalmente la presión arterial durante el Control Médico. Sin embargo, al $33.1 \%$ (49) de los no hipertensos nunca se les tomo la Presión Arterial durante el Control Médico. (Ver Gráfico No. 5)
Gráfico No. 5: Distribución de Frecuencia de Toma de Presión Arterial en el Control Médico en la Población Estudiada.

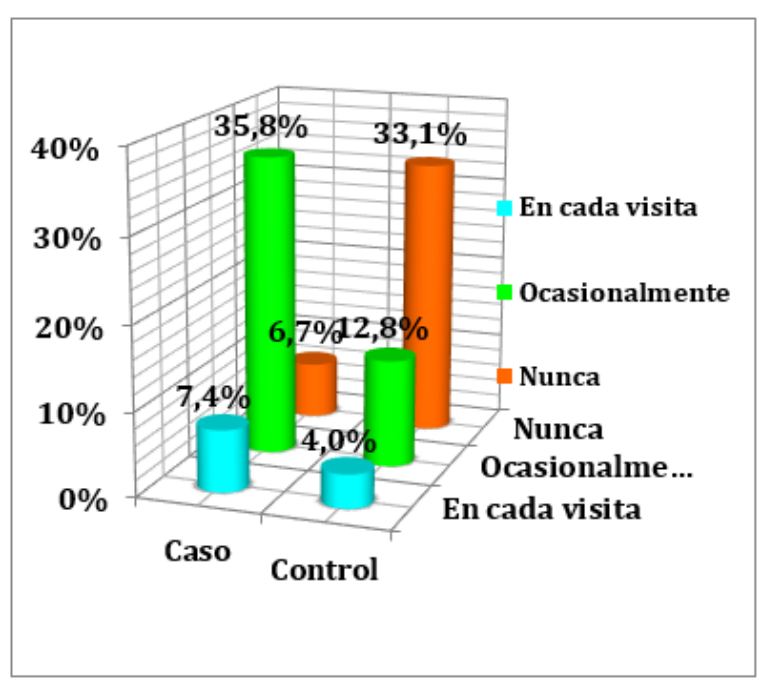

Fuente: Estudio de Factores de Riesgo Cardiovascular y Renal asociados a HTA en Pediatría.

De la población estudiada el $91.8 \%$ (68) la causa de la HTA es secundaria. Las principales etiologías de Hipertensión Arterial en la población estudiada fueron: Las Malformaciones de Vías Urinarias $29.5 \%$ (22), Glomerulonefritis 14.8\% (11), Síndrome Nefrítico $13.5 \% \quad(10)$, e Hipertensión Esencial $9.4 \% \quad$ (7). (Ver Gráfico No. 6). 
Gráfico No. 6: Distribución de la Etiología de Hipertensión Arterial en la Población Estudiada.

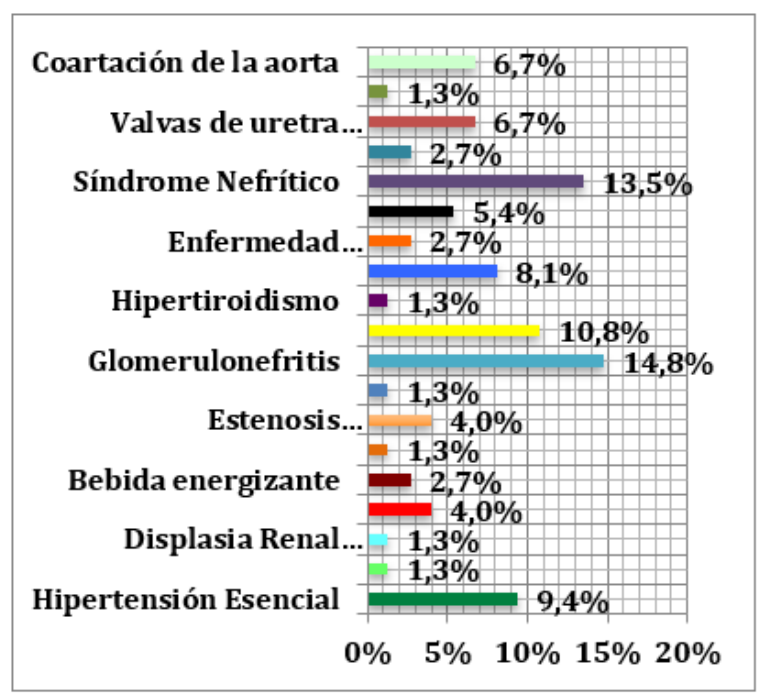

Fuente: Estudio de Factores de Riesgo Cardiovascular y Renal asociados a HTA en Pediatría.

Los Factores de Riesgo Cardiovascular y Renal Asociados a HTA en Pediatría identificados en el estudio fueron los siguientes (OR> 1 y Valor de $P<0.005)$ : los pacientes menores de 10 años de edad tiene una probabilidad de un $70 \%$ de tener menor riesgo HTA. El sexo masculino se asocia en 1.5 veces más riesgo y un bajo nivel académico se asocia en un $30 \%$ de riesgo. El antecedente familiar de HTA aumenta en casi 2 veces el riesgo, el antecedente familiar de Obesidad se asocia con el $10 \%$ de riesgo de desarrollar HTA, el consumo de alcohol incrementa en 10 veces el riesgo, el hábito de fumar aumenta en 11 veces el riesgo, el consumo de café aumenta en 2 veces el riesgo, la falta de ejercicio tiene un $49 \%$ de riesgo, el agregar Sal a los alimentos aumenta el riesgo en 3 veces. En relación a los antecedentes personales patológicos las enfermedades renales incrementan en 4 veces el riesgo, enfermedad autoinmunitaria tiene 40 veces mayor riesgo y en la enfermedad cardiaca el riesgo es del $10 \%$. (Ver Tabla No. 1 ).

Tabla 1. Distribución de las Variables relacionadas a Factores de Riesgo Cardiovascular y Renal asociadas a Hipertensión Arterial en Pediatría.

\begin{tabular}{|c|c|c|c|c|c|c|}
\hline \multirow[t]{2}{*}{ Variables } & \multirow[t]{2}{*}{ Hipertenso } & \multirow[t]{2}{*}{$\begin{array}{l}\text { No } \\
\text { Hipertenso }\end{array}$} & \multirow[t]{2}{*}{ OR } & \multicolumn{2}{|c|}{$\begin{array}{ll}\text { Intervalo } & \text { de } \\
\text { Confianza } & \text { del } \\
95 \% & \\
\end{array}$} & \multirow[t]{2}{*}{$P$} \\
\hline & & & & $\mathrm{NCl}$ & NCS & \\
\hline \multicolumn{7}{|l|}{ Edad } \\
\hline$<10$ años & 29 & 51 & 0.291 & 0.147 & 0.573 & 0.000 \\
\hline \multicolumn{7}{|l|}{ Sexo } \\
\hline Hombre & 45 & 37 & 1.552 & 0.808 & 2.979 & 0.186 \\
\hline \multicolumn{7}{|c|}{ Familiares con Antecedentes de Hipertensión Arterial } \\
\hline SI & 38 & 27 & 1.837 & 0.952 & 3.545 & 0.068 \\
\hline \multicolumn{7}{|c|}{ Antecedentes Familiares con Obesidad } \\
\hline SI & 12 & 11 & 1.109 & 0.455 & 2.700 & 0.821 \\
\hline \multicolumn{7}{|c|}{ Consumo de Alcohol } \\
\hline SI & 9 & 1 & 10.108 & 1.247 & 81.953 & 0.009 \\
\hline \multicolumn{7}{|c|}{ Hábito de Fumar } \\
\hline SI & 10 & 1 & 11.406 & 1.421 & 91.561 & 0.005 \\
\hline \multicolumn{7}{|c|}{ Consumo de Café } \\
\hline SI & 46 & 33 & 2.041 & 1.059 & 3.935 & 0.032 \\
\hline \multicolumn{7}{|c|}{ No realiza Ejercicio } \\
\hline SI & 17 & 28 & 0.490 & 0.239 & 1.004 & 0.049 \\
\hline \multicolumn{7}{|c|}{ Agrega Pisca de Sal } \\
\hline SI & 48 & 26 & 3.408 & 1.735 & 6.694 & 0.000 \\
\hline \multicolumn{7}{|c|}{ Antecedente Personal de Enfermedad Renal } \\
\hline SI & 54 & 30 & 3.960 & 1.983 & 7.909 & 0.000 \\
\hline \multicolumn{7}{|c|}{ Antecedente Personal de Enfermedad Cardíaca } \\
\hline SI & 10 & 41 & 0.126 & 0.056 & 0.282 & 0.000 \\
\hline \multicolumn{7}{|c|}{ Antecedente Personal de Enfermedad Autoinmune } \\
\hline SI & 26 & 1 & 39.542 & 5.192 & 30.145 & 0.000 \\
\hline \multicolumn{7}{|c|}{ Antecedente Personal de Diabetes Mellitus } \\
\hline SI & 2 & 1 & 2.028 & 0.180 & 22.860 & 0.560 \\
\hline \multicolumn{7}{|c|}{ Nivel de Escolaridad } \\
\hline $\begin{array}{l}\text { Primaria } \\
\text { Incompleta }\end{array}$ & 11 & 24 & 0.364 & 0.163 & 0,813 & 0.012 \\
\hline
\end{tabular}

Fuente: Estudio de Factores de Riesgo Cardiovascular y Renal asociados a HTA en Pediatría.

De acuerdo al análisis multivariado de la Regresión Logística Binaria la puntuación $\operatorname{Exp}(B)>1$, nos 
indica que a medida que el paciente vaya aumentando la edad, bajo nivel socioeconómico, vida sedentaria, tener antecedentes personales de enfermedad renal, cardíaca, tiroidea, cáncer y diabetes mellitus aumenta la probabilidad de presentar Hipertensión Arterial. La puntuación de Wald en el estudio indica que las variables (Edad (Wald:7.654;gl:1;P<0.05), Ejercicio (Wald:12.620;gl:1;P<0.001), Antecedentes Personales de Enfermedad Inmunitaria (Wald:4.329;gl:1;P<0.05)) aportan datos significativos a la predicción de presentar Hipertensión Arterial en Pediatría si existen Factores de Riesgo Renal y Cardiovascular. Además los valores se pueden generalizar a la población. (Ver Tabla No. 2).

Tabla 2. Modelo de Regresión Logística Binaria de las Variables relacionadas a Factores de Riesgo Cardiovascular y Renal asociadas a Hipertensión Arterial en Pediatría.

\begin{tabular}{|c|c|c|c|c|c|c|}
\hline \multirow[b]{2}{*}{ Variables } & \multirow[b]{2}{*}{ Wald } & \multirow[b]{2}{*}{$\begin{array}{l}\text { Grados de } \\
\text { Libertad } \\
\text { (gl) }\end{array}$} & \multirow[b]{2}{*}{$P$} & \multirow[b]{2}{*}{$\operatorname{Exp}(B)$} & \multicolumn{2}{|c|}{$\begin{array}{l}\text { Intervalo de Confianza } \\
95 \% \text { para Exp (B) }\end{array}$} \\
\hline & & & & & Inferior & Superior \\
\hline Edad > 10 años & 7.654 & 1 & 0.006 & 6.447 & 1.72 & 24.14 \\
\hline Sexo Masculino & 4.469 & 1 & 0.003 & 5.704 & 2.87 & 4.18 \\
\hline $\begin{array}{l}\text { Índice de Masa } \\
\text { Corporal }\end{array}$ & 0.049 & 1 & 0.825 & 0.892 & 0.825 & 0.892 \\
\hline $\begin{array}{l}\text { Bajo Nivel de } \\
\text { Escolaridad }\end{array}$ & 3.327 & 1 & 0.076 & 2.503 & 0.997 & 1.003 \\
\hline $\begin{array}{l}\text { Antecedentes } \\
\text { Familiares de }\end{array}$ & 8.649 & 1 & 0.004 & 8.442 & 0.104 & 0.442 \\
\hline
\end{tabular}

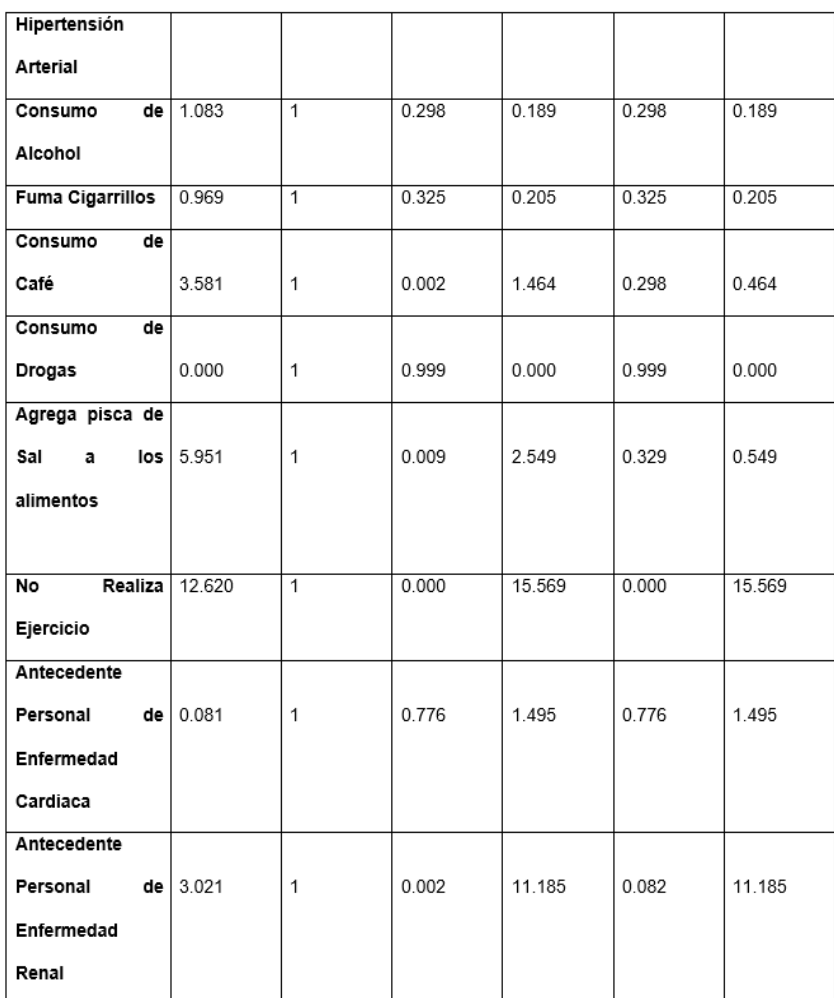

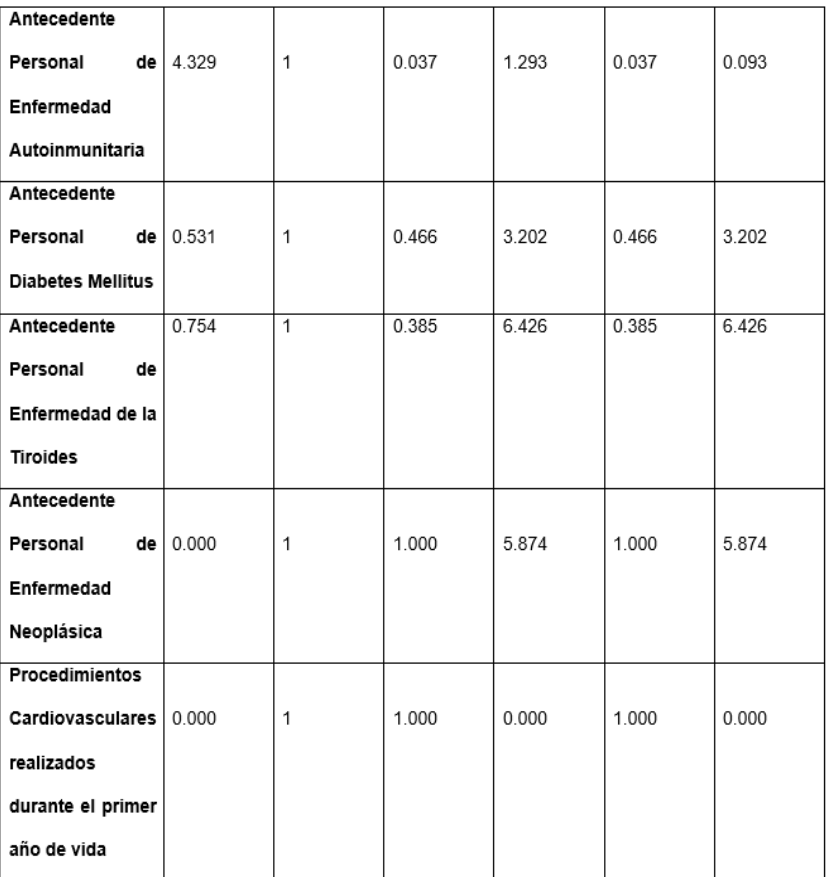

Fuente: Estudio de Factores de Riesgo Cardiovascular y Renal asociados a HTA en Pediatría. 


\section{DISCUSIÓN}

La Hipertensión Arterial (HTA) en la población pediátrica es un parámetro muy variable, presentando valores de normalidad que varían según el sexo y edad, aumentando progresivamente a lo largo de los años con el crecimiento y desarrollo corporal(15,16). En el mundo, las enfermedades cardiovasculares son responsables de aproximadamente 17 millones de muertes por año(1). Entre ellas, las complicaciones por la HTA causan 9,4 millones de muertes (2).

La HTA es la causa de por lo menos el $45 \%$ de las muertes por cardiopatías y el $51 \%$ de las muertes por enfermedades cardiovasculares ${ }^{(3)}$. La identificación de niños con HTA representa un desafío para los pediatras, el identificar y reconocer los factores de riesgo en etapas tempranas de la vida nos permitirá prevenir la HTA y así evitar las complicaciones tempranas de esta enfermedad(17). Los estudios nacionales relacionados con la HTA en pediatría no son frecuentes. El escaso conocimiento local sobre la epidemiologia y el impacto de cifras tensiónales a partir de edades tempranas es una interrogante que aún no tiene respuesta precisa, por la falta de estudios sobre los factores de riesgo de HTA en niños en la ciudad de San Pedro Sula. En la literatura nacional consultada no se encontró ningún estudio sobre HTA en Pediatría. En este trabajo de investigación hemos identificado 67 pacientes pediátricos con Hipertensión Arterial en el Instituto Hondureño de Seguridad Social-Hospital Regional del Norte lo que se traduce en una prevalencia de HTA según la distribución siguiente: $\quad 0.19 \%$ en Emergencia Pediátrica, $0.15 \%$ en Consulta Externa de Cardiología Pediátrica, $3.64 \%$ en Unidad de Cuidados Intensivos
Pediátricos, $0.25 \%$ en Sala de Pediatría A y $0.31 \%$ en Sala de Pediatría B y $0.24 \%$ en el IHSS-HRN. En Diálisis de HondurasSPS se identificaron 7 pacientes con HTA lo que traduce una prevalencia de $2.7 \%$. La prevalencia de la HTA es mayor entre los niños afroamericanos e hispanos en comparación con los niños blancos no hispanos, con tasas más altas entre los adolescentes que entre los niños más pequeños. La prevalencia de HTA en niños y adolescentes es de aproximadamente $3.5 \%,{ }^{(18,19)}$ con tasas más altas entre los niños y adolescentes con sobrepeso y obesidad que varía del $3.8 \%$ al $24.8 \%$ respectivamente ${ }^{(20)}$. Entre los pacientes con Insuficiencia Renal Crónica (IRC) el 50\% son conocidos por hipertensos ${ }^{(21-24)}$. En niños y adolescentes con enfermedad renal en etapa terminal (diálisis o trasplante), aproximadamente el $48 \%-79 \%$ son hipertensos, con $20 \%-70 \%$ con HTA incontrolada(25-29). Casi el $20 \%$ de la HTA pediátrica puede ser atribuida a IRC (30). Sin embargo, en nuestro medio la diferencia podría explicarse debido al infra diagnóstico de la HTA en pediatría. Estas cifras demuestran la importancia de la HTA como problema de salud pública y la necesidad de generar propuestas de políticas públicas saludables e investigación para prevenir las complicaciones desde edades tempranos y promover hábitos sanos desde la infancia. De los 74 casos identificados, el sexo masculino representa $60.8 \%$ (45), con una relación Hombres: Mujeres 1.5:1. La incidencia de HTA es consistentemente mayor en los varones (45-50\%) que en las niñas (35-40\%) (31). En el estudio el rango de edad fue de $11-15$ años $43.2 \%$ (32) con una media de 11.3 años. Se ha evidenciado que las cifras tensionales 
tienden a elevarse progresivamente con el crecimiento corporal, siendo en Pediatría la Hipertensión Secundaria la más frecuente en relación a los adultos que habitualmente es Primaria o Esencial(32). No se presentó ningún caso de HTA relacionado con Obesidad, el $56.7 \%$ (42) tenían un índice de Masa Corporal normal para la edad, sin embargo, la prevalencia creciente de la HTA se atribuye a la epidemia de obesidad, siendo mayor las cifras tensionales en este grupo de riesgo (33). El nivel de escolaridad de los pacientes con HTA, se encontró que el $49.9 \%$ (37) tenían un nivel académico superior a 6 años, sin embargo, estudios en pacientes con obesidad han evidenciado que un bajo nivel académico se asocia con hábitos dietéticos no saludables que conllevan a obesidad e hipertensión arterial (34-38).

En relación a los antecedentes familiares patológicos, las Enfermedades del Corazón representa el $41.8 \%$ (31) y de acuerdo al antecedente familiar de Hipertensión Arterial el 51.3\% (38) refirió tenerlo, sin embargo el $83.7 \%$ (62) no refirió tener el antecedente de Obesidad en la familia; cuando ambos padres son hipertensos, el $50 \%$ de los hijos heredará la condición, si solo es un padre el afectado, el riesgo es de $33 \%,(20,23)$ en este estudio no se determinó si uno 0 ambos padres era el que tenía el antecedente de HTA.

Según los hábitos tóxicos de la población estudiada no refirieron consumo de bebidas alcohólicas $87.8 \%$ (65), consumo de cigarrillos $86.5 \%$ (64) y consumo de drogas $97.3 \%$ (72); no obstante el consumo de café lo refirieron el $62.1 \%$ (46) de los pacientes con Hipertensión Arterial, la relación del consumo de café con las enfermedades cardiovasculares es controvertida ya que hay observaciones en ambos sentidos, la asociación ha sido objeto de una publicación basada en datos de cohortes del Health Prefessionals Followup Study y del Nurses Health Study en donde determinaron que una ingesta moderada de café (3 tazas/día) reduce la cifra de presión arterial, sin embargo, un consumo mayor a esta cifra se asocia a un efecto presor transitorio (4 horas tras la ingesta de café) con un aumento de 3-15 $\mathrm{mmHg}$ de la presión arterial sistólica (PAS) y unos $4-13 \mathrm{mmHg}$ de presión arterial diastólica respecto a las cifras basales (39). Se encontró que el sedentarismo estuvo presente en el $77.1 \%$ (57) de los pacientes con Hipertensión Arterial y el 62.2 (46) de los no hipertensos; los niños actualmente tienen malos hábitos de alimentación, pobre ejercicio físico y más ocio que los de generaciones anteriores, los resultados muestran que los niños que se mantienen sedentarios más de 2 horas al día (delante de la TV, ordenador, tablets y videoconsola), presentan un riesgo de $30 \%$ mayor de desarrollar HTA (40). En base a Agregar Pisca de Sal en los alimentos el $64.8 \%$ (48) de los pacientes con HTA respondieron afirmativa a esta interrogante; He $\mathrm{J}$ et al. Estudiaron en un ensayo aleatorio y cruzado, un total de 112 sujetos donde demostraron que una modesta reducción en la ingesta de sal, desde 12 a 6 gramos al día, disminuía la PAS hasta $10 \mathrm{mmHg}^{(24)}$. De los pacientes con HTA el $98.6 \%$ (73) refirieron que asisten a Control Médico, la frecuencia del Control Médico de "1 vez cada 6 meses" representa el $17.5 \%$ (26), la frecuencia de la toma de la Presión Arterial en el Control Médico fue "Ocasionalmente" $35.8 \%$ (53), y la toma de la Presión Arterial fue en la "Consulta de Seguimiento" 100\% (74) en los pacientes con Hipertensión Arterial y $98.6 \%$ (73) en los No Hipertensos, el rango de edad a la que le tomaron por primera vez la presión arterial fue entre los 11-15 años $36.4 \%$ (27); en relación a lo anterior queda evidenciado que la toma de 
la PA en los controles médicos no es de forma rutinaria ya que en esta población no suele ser una práctica habitual de la consulta médica y con la creciente prevalencia de HTA todo pediatra debe estar familiarizado con los manejos de las tablas de referencia de PA según la edad, sexo y talla;(34)la mitad de los niños yadolescentes entre 7-18 años nunca se les ha realizado un control de PA (23-27). En relación a los antecedentes personales de procedimientos cardiovasculares invasivos realizados durante el primer año el $98.7 \%$ (73) refirieron no tener antecedentes. El estadio I de Hipertensión Arterial al momento del diagnóstico representa $67.5 \% \quad(50)$, el $67.5 \% \quad$ (50) tenían más de 6 meses de diagnóstico de Hipertensión Arterial, el 70.2\% (52) usaba solo un antihipertensivo, el tipo de antihipertensivo más utilizado son los IECA 66.2\% (49). De acuerdo a la disminución de la Presión Arterial con el Ejercicio y la Dieta el $55.4 \% \quad$ (41) refirieron una disminución, de los cuales el $42.0 \%$ (31) no sabían cuántas cifras de Presión Arterial habían disminuido, el $39.1 \%$ (29) refirieron una disminución de $10 \mathrm{~mm} / \mathrm{Hg}$; la actividad física aeróbica constituye uno de los principales pilares del tratamiento no farmacológico en pacientes hipertensos, hay evidencias que demuestran que con la práctica regular de ejercicios aeróbicos (30-40 minutos de caminata, 3 o 4 veces por semana), puede haber reducción significativa (10 $\mathrm{mmHg}$ ) de la PAS y PAD, además de beneficios indirectos en la disminución de obesidad, estrés y niveles de ansiedad (34-37). En relación a los antecedentes personales patológicos más relevantes: el $72.9 . \% \quad$ (54) tenían antecedentes de enfermedad renal, el $13.5 \% \quad(10)$ enfermedad cardíaca, el $\quad 35.1 \% \quad$ (26) enfermedad autoinmunitaria. En relación a los antecedentes personales patológicos más relevantes: el $72.9 . \%$ (54) tenían antecedentes de enfermedad renal, el $13.5 \%$ (10) enfermedad cardíaca, el $35.1 \%$ (26) enfermedad autoinmunitaria. En relación a las enfermedades renales de los pacientes con HTA, fueron: Glomerulopatía $25.6 \%$ (19), Insuficiencia Renal Crónica $17.5 \%$ (13) y Malformaciones de Vías Urinarias $12.1 \%$ (9). Sin embargo, de las enfermedades cardiacas de los pacientes con HTA, en orden de frecuencia: Coartación de la aorta $6.7 \%$ (5), Insuficiencia aortica $2.7 \%$ (2) y Fístulas Aurículo-Ventriculares $1.3 \%$ (1). La HTA Secundaria representa $91.8 \%$ (68), siendo las etiologías encontradas en el estudio: Malformación de vías urinarias 29.5\% (22), Glomerulonefritis $14.8 \%$ (11) y Síndrome nefrítico 13.5\% (10); en general, cuanto menor es la edad del niño y mayor sean los valores de PA, más probable es que la HTA sea de causa secundaria, en pediatría predomina la causa secundaria en la mayoría de los casos con HTA, las causas van a variar de acuerdo a la edad del paciente, siendo en menores de 1 mes las lesiones renales congénitas, trombosis de la arterial renal y coartación de la aorta entre las causas más destacadas, sin embargo, en niños de 1 mes a 6 años predominan la enfermedad parenquimatosa renal (glomerulonefritis, cicatrices renales, enfermedad poliquística) y renovascular, en niños mayores de 10 años ya comienza a aparecer la HTA esencial y enfermedades parenquimatosas renal.

De acuerdo al seguimiento en Consulta Externa de los pacientes con HTA: el 90.5\% (67) fueron referidos a la Consulta Externa de Nefrología el $77.0 \%$ (57) fueron referidos a la Consulta Externa de Cardiología, y el $41.8 \%$ (31) fueron referidos a la Consulta Externa de Nutrición.En cuanto a los factores de riesgo encontrados en el estudio los hallazgos fueron: los niños 
menores de 10 años de edad tienen menor riesgo de HTA en un $70 \%$, el sexo masculino se asoció en 1.5 veces más de padecer HTA, el antecedente familiar de HTA aumenta 2 veces el riesgo de HTA, el consumo de alcohol y el hábito de fumar incrementa en 10 y 11 veces el riesgo de HTA respectivamente, el consumo de café y agregar sal a los alimentos aumenta en 2 veces el riesgo de HTA respectivamente. El sedentarismo incrementa en $49 \%$ el riesgo de HTA. De acuerdo a los antecedentes personales patológicos, las enfermedades renales aumentan en 4 veces el riesgo, en las auto inmunes el riesgo es 40 veces mayor, y en la enfermedad cardíaca el riesgo es del $10 \%$.

De acuerdo a los resultados de la Regresión Logística Binaria, en la Primera Fase, en el estudio se pudo determinar que los factores de riesgo renales y cardiovasculares tienen una probabilidad del $100 \%$ de acierto de que se asocien con HTA y las variables que tuvieron significancia estadística son: Edad ( $P$ <0.001), Escolaridad $(P=0.012)$, Consumo de Alcohol ( $P=0.009)$, Fuma Cigarrillos ( $P=0.005)$, Consumo de Café $(P$ $=0.032)$, Agrega Pisca de Sal a los alimentos ( $P<0.001)$, Antecedentes Personales de Enfermedad Renal ( $P<0.001)$, Antecedentes Personales de Enfermedad Cardiaca ( $P$ $<0.001)$, Antecedentes Personales de Enfermedad Autoinmunitaria $(P<0.001)$, No realiza Ejercicio $(P=0.049)$. En la Segunda Fase de la Regresión Logística se pudo determinar que hay un $86.5 \%$ de probabilidad de que el paciente tenga HTA si hay un factor de riesgo renal 0 cardiovascular asociado, con una Especificidad del $83.8 \%$ y una Sensibilidad de $89.2 \%$. En relación a la puntuación $\operatorname{Exp}(B)$ nos indicó que a medida que el paciente vaya aumentando la edad, un Bajo Nivel Académico llevar una vida sedentaria, tener Antecedentes Personales de Enfermedad, Renal, Cardiaca, Enfermedad de la Tiroides, Cáncer y Diabetes Mellitus hay mayor probabilidad de que presente HTA. La puntuación de Wald en el estudio nos indicó que la variables (Edad (Wald:7.654;gl:1;P<0.05), Falta de Ejercicio (Wald:12.620;gl:1;P<0.001), Antecedentes Personales de Enfermedad Inmunitaria (Wald:4.329;gl:1;P<0.05)) aportan datos significativos a la predicción de presentar Hipertensión Arterial en Pediatría si existen Factores de Riesgo Renal y Cardiovascular, además los valores se pueden generalizar a la población.

En conclusión, Los Factores de Riesgo Cardiovascular y Renal asociados a Hipertensión Arterial en Pediatría se relacionan con el tipo de estilo de vida del paciente el cual puede ser modificado en edades tempranas para disminuir el riesgo cardiovascular en la edad adulta.

Se recomienda: Diseñar estrategias a nivel Nacional y Regional desde la atención primaria con actividades relacionadas a programas preventivo-promocionales dirigidos a estilos de vida saludables que disminuyan los factores de riesgo asociados a la Hipertensión Arterial. 


\section{REFERENCIAS BIBLIOGRÁFICAS}

1. Litwin Mieczysław, Kułaga Zbigniew. Pediatric hypertension: Definition, normative values, epidemiology, screening and treatment. Postępy Nauk Medycznych. [Revista en internet] 2015 [Citado, 13 febrero 2016]; (28):(11): 325-45. Disponible en: $\quad$ http://www.pnmedycznych.pl/wpcontent/uploads/2016/01/ pnm_2015_787-793.pdf

2. OMS [Internet]. Organización Mundial de la Salud. Información General sobre la Hipertensión en el mundo. 2013. [Actualizado en 2013; consultado 15 Mayo 2016]. Disponible en: www.who.int/about/ licensing/copyright_form/en/index.html

3. Barba G, Buck C, Bammann $\mathrm{K}$ et al.; IDEFICS consortium: Blood pressure reference values for European nonoverweight school children: the IDEFICS study. Int J Obes (Lond). [Revista en internet] 2014 [Citado, 20 febrero 2016] ;38: (2): 48-56. Disponible en: http:// www.ncbi.nlm.nih.gov/pubmed/25219411

4. Dereziński T, Kułaga Z, Litwin M: Prevalence of arterial hypertension and anthropometrical predictors of elevated blood pressure in 14 years old adolescents. European Society of Hypertension Meeting 2015, Milan, abstract.

5. Mozaffarian D, Benjamin EJ, Go AS, et al; Writing Group Members; American Heart Association Statistics Committee; Stroke Statistics Subcommittee. Executive summary: heart disease and stroke statistics-2016 update: a report from the American Heart Association. Circulation. [Revista en internet] 2016 [Citado, 21 abril 2018];133(4):447-454. Disponible en: https://www.ncbi.nlm.nih.gov/ pubmed/26673558
6. Daley MF, Sinaiko AR, Reifler LM et al.: Patterns of Care and Persistence After Incident Elevated Blood Pressure. Pediatrics. [Revista en internet] 2013 [Citado, 25 febrero 2016]; 132: e349e355. Disponible en: http:// www.ncbi.nlm.nih.gov/pubmed/23821694.

7. Kit BK, Kuklina E, Carroll MD, Ostchega Y, Freedman DS, Ogden CL. Prevalence of and trends in dyslipidemia and blood pressure among US children and adolescents, 1999-2012. JAMA Pediatr. [Revista en internet] 2015 [Citado, 21 abril 2018];169(3):272-279. Disponible en: https://www.ncbi.nlm.nih.gov/ pubmed/25599372

8. Hansen ML, Gunn PW, Kaelber DC. Underdiagnosis of hypertension in children and adolescents. JAMA. [Revista en internet] 2007 [Citado, 21 abril 2018]; 298(8):874-879. Disponible en: https:// www.ncbi.nlm.nih.gov/pubmed/17712071.

9. 9. McNiece $\mathrm{KL}$, Poffenbarger TS, Turner JL, Franco KD, Sorof JM, Portman RJ. Prevalence of hypertension and prehypertension among adolescents. J Pediatr. [Revista en internet] 2007 [Citado, 21 abril 2018];150(6):640-644, 644.e1. Disponible en: https:// www.ncbi.nlm.nih.gov/pubmed/17517252

10. Martínez HJ, Reyes HJ, Thiebaud AM, Gomez O. Prevalencia de hipertensión arterial en adultos de El Progreso. Rev Med Hondur. [Revista en internet] 2005 [Citado, 20 abril 2016]; 73:60-64. Disponible en: http:// cidbimena.desastres.hn/RMH/pdf/2005/ pdf/Vol73-2-2005-2.pdf 
11. Hernández CPK, Zambrano IL. Prevalencia de Hipertensión Arterial en una Comunidad Urbana de Honduras. Rev. Fac. Cienc. Med. [Revista en internet] 2009 [Citado, 20 abril 2016; 70:40-48. Disponible en: http://cidbimena.desastres.hn/ RFCM/pdf/2009/pdf/

12.

Flynn JT, Kaelber DC, Baker-Smith $\mathrm{CM}$, et al. Clinical Practice Guideline for Screening and Management of High Blood Pressure in Children and Adolescents. Pediatrics. [Revista en internet] 2017 [Citado, 10 abril 2018];140(3):e20171904. Disponible en: http://pediatrics.aappublications.org/

13. content/140/3/e20171904. figures-only

de Moraes AC, Carvalho HB, Siani A et al.; IDEFICS consortium: Incidence of high blood pressure in children - effects of physical activity and sedentary behaviors: the IDEFICS study: High blood pressure, lifestyle and children. Int $\mathrm{J}$ Cardiol. [Revista en internet] 2015 [Citado, 10 febrero 2016];180:

14. (23):165-701. Disponible en: http:// www.ncbi.nlm.nih.gov/

pubmed/25460372.

Dionne JM, Abitbol CL, Flynn JT. Hypertension in infancy: diagnosis, management and outcome [published correction appears in Pediatr Nephrol. [Revista en internet] 2012 [Citado, 15 marzo 2018];27(1):159-60]. Pediatr Nephrol. 2012;27(1):17-32 Disponible en: https://www.ncbi.nlm.nih.gov/ pubmed/21258818

15. Kent AL, Chaudhari T. Determinants of neonatal blood pressure. Curr Hypertens Rep. [Revista en internet] 2013 [Citado, 16 marzo 2018]; 15(5):426-432. Disponible en: https://www.ncbi.nlm.nih.gov/ pubmed/23917808

16. Diabetes Care. [Internet]. Pyle Laura. Today Study Group: Rapid rise in hypertension and nephropathy in youth with type 2 diabetes. Diabetes Care. 2013; 36: 1735-1741. [Actualizado Junio 2013, citado 20 febrero 2016] Disponible en: http:// care.diabetesjournals.org/ content/36/6/1735

17.

National High Blood Pressure Education Program Working Group on High Blood Pressure in Children and Adolescents. The fourth report on the diagnosis, evaluation, and treatment of high blood pressure in children and adolescents. Pediatrics. [Revista en internet] 2004 [Citado, 15 diciembre 2017];114(2, suppl 4th Report):555-576 Disponible en: https:// catalog.nhlbi.nih.gov/sites/default/files/ publicationfiles/05-5267.pdf

18.

Sorof J, Daniels S. Obesity hypertension in children: a problem of epidemic proportions. Hypertension. [Revista en internet] 2002 [Citado, 5 enero 2018];40(4):441-447. Disponible en: https://www.ncbi.nlm.nih.gov/

19. pubmed/12364344

Sorof JM, Lai D, Turner J, Poffenbarger T, Portman RJ. Overweight, ethnicity, and the prevalence of hypertension in school-aged children. Pediatrics. [Revista en internet] 2004 [Citado, 5 febrero 2018];113(3, pt 1):475-482. Disponible en: https:// www.researchgate.net/ publication/5848894_Sorof_JM_Lai_D_Tur ner_J_Poffenbarger_T_Portman_RJ_Ōver weight_ethnicity_and_the_prevalence_of_h ypertension_in_school-aged_children 
20. Koebnick C, Black MH, Wu J, et al. High blood pressure in overweight and obese youth: implications for screening. J Clin Hypertens (Greenwich). [Revista en internet] 2013 [Citado, 7 febrero 2018];15(11):793-805. Disponible en: https://www.ncbi.nlm.nih.gov/pmc/articles/ PMC3849231/

21. Falkner B, Gidding SS, Ramirez-Garnica G, Wiltrout SA, West D, Rappaport EB. The relationship of body mass index and blood pressure in primary care pediatric patients. J Pediatr. [Revista en internet] 2006 [Citado, 7 noviembre 2017]; 148(2):195-200. Disponible en: https:// www.ncbi.nlm.nih.gov/pubmed/16492428

22. Lurbe E, Invitti C, Torro I, et al. The impact of the degree of obesity on the discrepancies between office and ambulatory blood pressure values in youth [published correction appears in $\mathrm{J}$ Hypertens. 2007;25(1):258]. J Hypertens. [Revista en internet] 2006 [Citado, 10 noviembre 2017];24(8):1557-1564. Disponible en: https:// www.ncbi.nlm.nih.gov/pmc/articles/ PMC4478325/

23. Skinner AC, Perrin EM, Moss LA, Skelton JA. Cardiometabolic risks and severity of obesity in children and young adults. N Engl J Med. [Revista en internet] 2015 [Citado, 10 marzo 2018];373(14):13071317. Disponible en: http://www.nejm.org/ doi/full/10.1056/NEJMoa1502821

24.T.r.k K, P.lfi A, Szel.nyi Z, Moln.r D. Circadian variability of blood pressure in obese children. Nutr Metab Cardiovasc Dis. [Revista en internet] 2008 [Citado, 12 marzo 2018];18(6):429-435. Disponible en: https://www.ncbi.nlm.nih.gov/ pubmed/18063354
25. Framme J, Dangardt F, M.rild S, Osika W, W.hrborg P, Friberg P. 24-h systolic blood pressure and heart rate recordings in lean and obese adolescents. Clin Physiol Funct Imaging. [Revista en internet] 2006 [Citado, 12 abril 2018];26(4):235-239. Disponible en: https:// www.ncbi.nlm.nih.gov/ pubmed/16836697

26. Westerst.hl M, Marcus C. Association between nocturnal blood pressure dipping and insulin metabolism in obese adolescents. Int J Obes. [Revista en internet] 2010 [Citado, 13 abril 2018] ;34(3):472-477. Disponible en: https://www.ncbi.nlm.nih.gov/ pubmed/19752880

27. Macumber IR, Weiss NS, Halbach SM, Hanevold CD, Flynn JT. The association of pediatric obesity with nocturnal nondipping on 24-hour ambulatory blood pressure monitoring. Am J Hypertens. [Revista en internet] 2016 [Citado, 13 febrero 2018];29(5):647-652. Disponible en: https:// www.ncbi.nlm.nih.gov/ pubmed/26310663

28. Archbold KH, Vasquez MM, Goodwin JL, Quan SF. Effects of sleep patterns and obesity on increases in blood pressure in a 5-year period: report from the Tucson Children's Assessment of Sleep Apnea Study. J Pediatr. [Revista en internet] 2012 [Citado, 12 diciembre 2017];161(1):26-30. Disponible en: https://www.ncbi.nlm.nih.gov/ pubmed/22284918 
29. Javaheri S, Storfer-Isser A, Rosen CL, Redline S. Sleep quality and elevated blood pressure in adolescents. Circulation. [Revista en internet] 2008 [Citado, 10 enero 2017]; 118(10):10341040. Disponible en: https:// www.ncbi.nlm.nih.gov/pubmed/18711015

30.

Au CT, Ho CK, Wing YK, Lam HS, Li AM. Acute and chronic effects of sleep duration on blood pressure. Pediatrics. [Revista en internet] 2014 [Citado, 10 septiembre 2017]; 133(1). Disponible en: www.pediatrics.org/cgi/content/full/133/1/ e64

31. Flynn JT, Mitsnefes M, Pierce C, et al. Chronic Kidney Disease in Children Study Group. Blood pressure in children with chronic kidney disease: a report from the Chronic Kidney Disease in Children study. Hypertension. [Revista en internet] 2008 [Citado, 13 octubre 2017];52(4):631-637. Disponible en: https://www.ncbi.nlm.nih.gov/ pubmed/18725579

32. Samuels J, Ng D, Flynn JT, et al. Chronic Kidney Disease in Children Study Group. Ambulatory blood pressure patterns in children with chronic kidney disease. Hypertension. [Revista en internet] 2012 [Citado, 03 abril 2017]; 60(1):43-50. Disponible en: https:// www.ncbi.nlm.nih.gov/pubmed/22585950

33. Shatat IF, Flynn JT. Hypertension in children with chronic kidney disease. Adv Chronic Kidney Dis. [Revista en internet] 2005 [Citado, 07 noviembre 2017];12(4):378-384. Disponible en: https://www.ncbi.nlm.nih.gov/pmc/articles/ PMC5538700/
34. 34.Chavers BM, Solid CA, Daniels FX, et al. Hypertension in pediatric longterm hemodialysis patients in the United States. Clin J Am Soc Nephrol. [Revista en internet] 2009 [Citado, 10 noviembre 2017];4(8):1363-1369. Disponible en: http://cjasn.asnjournals.org/ content/4/8/1363.full

35. Seeman T. Hypertension after renal transplantation. Pediatr Nephrol. [Revista en internet] 2009 [Citado, 12 enero 2018];24(5):959-972. https:// www.ncbi.nlm.nih.gov/pubmed/17955265.

36. Tkaczyk M, Nowicki M, BałaszChmielewska I, et al. Hypertension in dialysed children: the prevalence and therapeutic approach in Poland-a nationwide survey. Nephrol Dial Transplant. [Revista en internet] 2006 [Citado, 15 febrero 2018];21(3):736-742. Disponible en: https://academic.oup.com/ ndt/article/21/3/736/1854588

37. Kramer AM, van Stralen KJ, Jager KJ, et al. Demographics of blood pressure and hypertension in children on renal replacement therapy in Europe. Kidney Int. [Revista en internet] 2011 [Citado, 08 marzo 2018];80(10):1092-1098. Disponible en: https:// www.sciencedirect.com/science/article/pii/ S008525381554951X

38. Halbach SM, Martz K, Mattoo T, Flynn $\mathrm{J}$. Predictors of blood pressure and its control in pediatric patients receiving dialysis. J Pediatr. [Revista en internet] 2012 [Citado, 15 marzo 2018];160(4):621-625.e1. Disponible en: https://www.ncbi.nlm.nih.gov/ pubmed/22056352 
39. Kaelber DC. IBM explorys cohort discovery tool. Disponible en: www.ibm.com/ watson/ health/ explorys. Acceso en 21 marzo 2018.

40. de Simone G, Devereux RB, Daniels SR, Koren MJ, Meyer RA, Laragh JH.

Effect of growth on variability of left ventricular mass: assessment of allometric signals in adults and children and their capacity to predict cardiovascular risk. J Am Coll Cardiol. [Revista en internet] 1995 [Citado, 03 abril 2018]; 25(5):1056-1062. Disponible en: https:// www.ncbi.nlm.nih.gov/pubmed/7897116 